\title{
THE OUTCOME OF LIMBERG FLAP RECONSTRUCTION PROCEDURE FOR PRIMARY NATAL CLEFT PILONIDAL SINUS DISEASE: FOUR YEARS' EXPERIENCE AT A TEACHING HOSPITAL IN PAKISTAN
}

\author{
WASIF MAJEED CHAUDHRY ${ }^{1}$, AFZA SALEEM ${ }^{1}$, MARYAM JAMIL ${ }^{1}$, MUHAMMAD SADDIQUE ZISHAN ${ }^{1}$, \\ ADNAN SADIQ BUTT ${ }^{2}$, MUHAMMAD AKRAM DOGAR ${ }^{3}$ \\ ${ }^{1}$ Department of General surgery, Lahore Medical and Dental College/ Ghurki Trust Teaching Hospital, Lahore, \\ ${ }^{2}$ Department of General surgery, Lahore General Hospital, Lahore, \\ ${ }^{3}$ Department of General Surgery, Central Park Medical College, Lahore.
}

\begin{abstract}
Background: Natal cleft pilonidal sinus disease is known for its high recurrence rate after surgery. Limberg flap reconstruction procedure has been used by many surgeons as their primary choice owing to its low recurrence rate and less postoperative complications.

Objective: To assess the outcome of Limberg flap reconstruction procedure in patients with primary natal cleft pilonidal sinus disease.

Methods: This descriptive case series was conducted at Ghurki trust teaching hospital, Lahore, Pakistan, between January 2015 to December 2018. 110 consecutive patients with primary natal cleft pilonidal sinus disease were included in this study. The evaluated outcomes of this procedure included duration of hospital stay, postoperative pain, primary healing, surgical site Infection, seroma formation, flap necrosis and, recurrence.

Results: The patients age ranged between 18 years to 60 years. The mean age was $26.6 \pm 8.6$ years. 73 patients $(66.36 \%)$ were males and 23 patients (33.63\%) were females. The duration of disease ranged between 1 month to 6 months with a mean of $3.7 \pm 1.7$ months. The mean operating time was $47.50 \pm 5.14$ minutes. The mean duration of hospital stay was $1.87 \pm 0.80$ days. The mean postoperative pain score calculated by the visual analogue scale (VAS) twenty-four hours after the surgery was 3.45 .95 patients $(86.36 \%)$ had complete primary healing without any complication. 9 patients $(8.18 \%)$ had a surgical site infection and 6 patients $(5.45 \%)$ had seroma formation. No patient suffered from flap necrosis. 2 patients $(1.81 \%)$ who had complete primary healing presented with recurrence of the disease within six months of the procedure with a period of one year of follow-up designated for every patient.

Conclusion: Limberg flap reconstruction procedure is a safe and efficacious treatment option for primary natal cleft pilonidal sinus disease because it provides early complete primary healing in most patients, and shortens the duration of hospital stay thus reducing the cost of treatment. The pain scores fall in the moderate post-operative pain score category and the complication rates and the recurrence rates are very low.
\end{abstract}

Key words: Natal Cleft, Pilonidal Sinus Disease, Limberg flap reconstruction procedure

How to cite this article: Chaudhry WM, Saleem A, Jamil M, Zishan MS, Butt AS, Dogar MA. The outcome of Limberg Flap reconstruction procedure for Primary Natal Cleft Pilonidal Sinus Disease: Four years' experience at a teaching hospital in Pakistan. Pak Postgrad Med J 2020;31(3): 123-127

This is an Open Access article distributed under the terms of the Creative Commons Attribution License (http://creativecommons.org/licenses/by/3.0), which permits unrestricted use, distribution, and reproduction in any medium, provided the original work is properly cited.

DOI: https://doi.org/10.51642/ppmj.v31i03.331

Correspondence to: Wasif Majeed Chaudhry,

Associate Professor,

Department of General surgery, Lahore Medical and

Dental College/ Ghurki trust teaching hospital

Lahore, Pakistan.

Email: drwasifchaudhry@gmail.com

\section{INTRODUCTION}

Natal cleft pilonidal sinus disease is a blind epithelial tract which contains hair and is found most commonly in the skin of the natal cleft, a few inches above the external anal opening. ${ }^{1,2}$ This disease is more common in young hairy males but the age range varies. ${ }^{1,2}$ Its presentation is variable in the form of an abscess or a discharging sinus oozing out pus or blood. ${ }^{3}$ This disease effects men more 
than women. ${ }^{4}$ Karydakis proposed three main causative factors for the natal cleft pilonidal sinus disease, namely, the abundant broken hair from the nape of the neck and back, the excessive force causing these hair to impact in the skin and the susceptibility of the skin to infection due to a deep natal cleft.$^{4-5}$ A deep natal cleft acts as a nidus for penetration of hair causing bacterial contamination. ${ }^{3,4}$ Chronic itching, sedentary life style, family history, poor hygiene and excessive hairiness are other major etiological factors for this disease ${ }^{3,4,6}$. The broken hair move inwards, roll up and form a hair nest inside the skin of the natal cleft causing pain and infection. ${ }^{7}$

The surgical treatment of primary natal cleft pilonidal sinus disease is variable from wide excision followed by healing with secondary intention to excision followed by reconstruction with a flap. ${ }^{3}$ A standard surgical treatment option has not yet been established. ${ }^{3}$ Wound infection, seroma formation, and high recurrence rate are common with almost all surgical procedures. ${ }^{1}$ Flap reconstruction procedures eliminate the etiology of the pilonidal sinus disease by flattening the inter gluteal sulcus. ${ }^{8}$ Limberg flap procedure is a reconstruction technique which utilizes the superior gluteal and sacral perforators for reconstruction. ${ }^{4}$ Some studies show that Limberg flap rotation procedure has better results as compared to the other procedures like, Karydakis and Bascom techniques in terms of shorter hospital stay, early return to work, lower pain scores, high patient satisfaction, significantly lower frequency of wound infection and seroma formation, better complete healing duration and lower recurrence rates for sacrococcygeal pilonidal sinus disease. ${ }^{1,9-10}$ While other studies show that karydakis flap was better than the Limberg flap procedure in terms of significantly shorter mean length of hospital stay, lower pain score, lower recurrence rate and lower complication rate. ${ }^{7}$ Therefore, the literature shows conflicting opinion regarding the outcomes of different flap reconstruction procedures for primary sacrococcygeal pilonidal sinus disease. This research was planned to evaluate the outcomes of Limberg flap reconstruction procedure at a teaching hospital. This study will generate more data and will provide evidence to the surgeons for selecting an appropriate surgical procedure for the treatment of primary sacrococcygeal pilonidal sinus disease in future practice. The objective of this study was to assess the outcome of Limberg flap reconstruction procedure in patients with primary natal cleft pilonidal sinus disease. The evaluated outcomes of this procedure included duration of hospital stay, postoperative pain score, primary healing, surgical site Infection, seroma formation, flap necrosis and, recurrence.

\section{METHODS}

This descriptive case series was conducted at Ghurki trust teaching hospital, Lahore, Pakistan between January 2015 to December 2018. Ethical approval for this research was taken from the institutions ethical review board (ERB). The first patient was enrolled on $10^{\text {th }}$ of January 2015 and the last patient was enrolled on $20^{\text {th }}$ December 2017. The last follow-up visit of the last enrolled patient was done on $20^{\text {th }}$ December, 2018. 110 consecutive patients presenting in the outpatient department who fulfilled the inclusion criteria were counseled and explained in detail about this study. Patients who were willing to give informed consent for Limberg flap procedure and whose ages were between 18 to 60 years and presented with primary sacrococcygeal pilonidal sinus disease without abscess formation and infection were selected. Patients who were declared unfit to undergo general Anesthesia by the Anesthesia team due to comorbidities were excluded from this study. Patients who had recurrent natal cleft pilonidal sinus disease, uncontrolled diabetes mellitus, uncontrolled hypertension, renal failure, and immunosuppression were also excluded from this study.

All the data was recorded on a predesigned proforma. All the patients detailed history was taken by one surgical resident and their clinical examination was performed by one consultant surgeon. All patients had their laboratory investigations done which were required by the anaesthesia team for general anaesthesia. Informed consent was taken from every patient. All patients underwent Limberg flap reconstruction procedure. All the procedures were performed by three consultant surgeons from one surgical team who were trained to do this procedure and all the pre- and post-operative patient's evaluation were done by the same three surgeons to eliminate bias.

The trimming of hair from the sacrococcygeal area was done on table just before the surgery. All patients were given 1.2-gram, intravenous Amoxicillin with Clavulanic acid and $400 \mathrm{mg}$ intravenous Metronidazole at induction of anaesthesia. All these surgeries were performed under general anesthesia in the prone, jackknife position. A sterile skin marker was used to mark the Limberg flap as shown in Figure.1. ${ }^{11}$ First a line was drawn from point $A$ to point $C$ vertically in the midline including all the sinus orifices and tracts and its length was measured (Figure . 1.) The line between point B to point $\mathrm{D}$ transected the midpoints of the line between point $\mathrm{C}$ to point $\mathrm{A}$ at right angles and was $60 \%$ of its length. ${ }^{11}$ This ratio of these lengths determined the correct shape of the rhomboid. The flap line was then marked between Point $\mathrm{D}$ to point $\mathrm{E}$ which was a direct continuation of the line between point $\mathrm{B}$ to point $\mathrm{D}$ and was of equal length from the point $B$ to point $C$, to which it was sutured after rotation. Point $\mathrm{E}$ to point $\mathrm{F}$ were 
parallel to point $\mathrm{D}$ and point $\mathrm{A}$ and were of equal length and after rotation, it was sutured to points $C$ to point $D$ (Figure. 1). ${ }^{11}$ Rhomboid shaped excision( Point A,B,C,D) was performed up to the fascia overlying the sacrum and a Limberg flap was rotated preserving its blood supply and sutured subcutaneously including fat and fascia with Vicryl 3/0 suture and the skin was sutured with interrupted mattress polypropylene 4/0 suture (Figure. 2.). Suction drain was placed in all the patients in the subcutaneous plane. Intravenous antibiotics were continued in every patient for 48 hours postoperatively and continued orally for another 5 days postoperatively. The operating time was calculated for every patient in minutes. Patients were discharged when the discharge criteria were met and length of hospital stay was recorded in hours. All the patient's data was recorded on the proforma which included the operating time in minutes, the duration of hospital stay in days, the postoperative pain score calculated by VAS at 24 hours after the procedure, flap necrosis, surgical site infection and seroma formation.

Patients were followed in the outpatient's department on the seventh and 14 days after the surgery. Wound infection was recorded by looking for redness, erythema and pus discharge from the wound site. The third follow-up visit was done at six months interval from the surgery to look for recurrence by looking for a recurrent sinus in the natal cleft and any history of pus or blood discharge from the wound on clinical examination. The fourth follow-up visit was done at one year to record the recurrence of the pilonidal sinus by looking at the same parameters seen for recurrence on the third followup visit. Some patients did not come for follow-up on their own and they were called and reminded to come for the follow-up visit to record any complications and recurrence.

Figure.1. Pre-operative marking of Limberg Flap

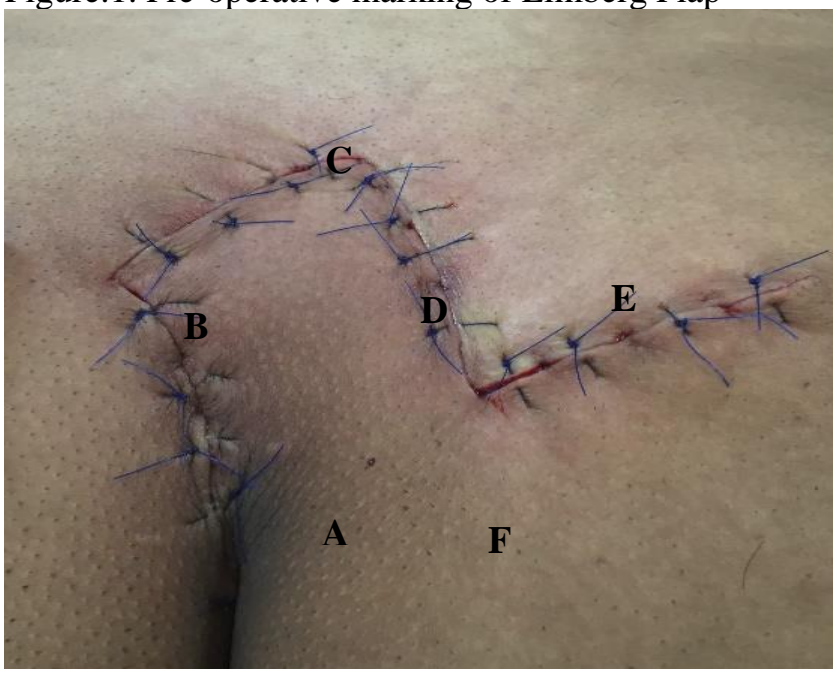

Figure.2. Scar after skin stitching

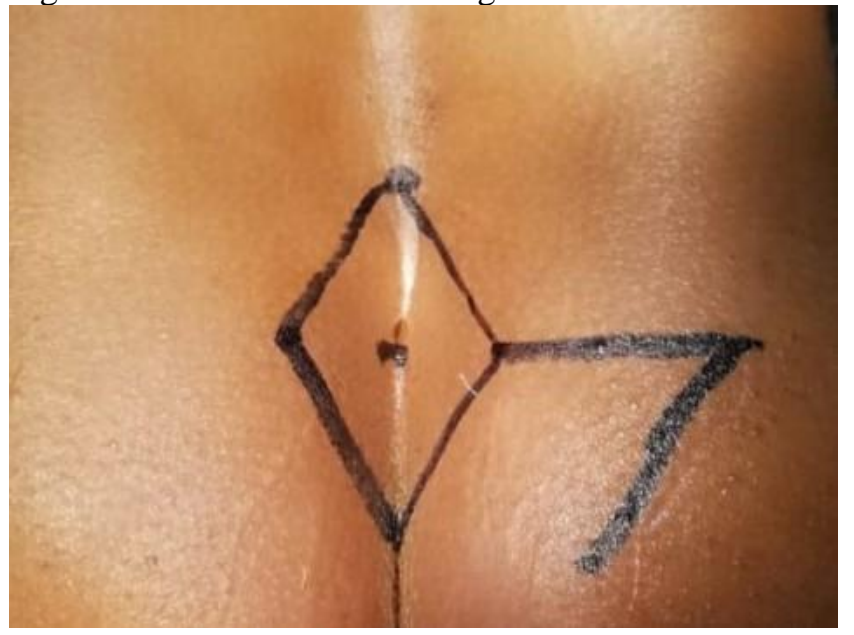

The SPSS version 21.0 was used for the analysis of the collected data. Numerical variables i.e. age, duration of disease, operating time and length of hospital stay are presented by mean $\pm \mathrm{SD}$. Categorical variables i.e. gender, wound infection and seroma formation are presented as frequency and percentages.

\section{RESULTS}

A total of 110 patients underwent Limberg flap procedure in this study. The patients age ranged between 18 years to 60 years. The mean age was $26.6 \pm 8.6$ years. 73 patients $(66.36 \%)$ were males and 37 patients (33.63\%) were females as shown in Table. 1 . The disease duration ranged between 1 month to 6 months. The mean disease duration was $3.7 \pm 1.7$ months. The mean operating time was $47.50 \pm 5.14$ minutes. The mean duration of hospital stay was $1.87 \pm 0.80$ days. The mean postoperative pain score calculated by VAS, twenty-four hours after the surgery was 3.45 .95 patients $(86.36 \%)$ had complete primary healing without any complication (Table.2). 9 patients $(8.18 \%$ ) had a surgical site infection and 6 patients $(5.45 \%)$ had seroma formation. No patient suffered from flap necrosis. 2 patients $(1.81 \%)$ who had complete primary healing presented with recurrence of the disease within six months of the procedure with a period of one year of follow-up designated for every patient .

Table .1. Age, gender distribution and duration of disease

\begin{tabular}{lc}
\hline Characteristics & $\mathrm{n}=110$ \\
Age (years) & $26.6 \pm 8.6$ \\
Gender & \\
$\quad$ Male & $73(66.36 \%)$ \\
$\quad \bullet$ Female & $37(33.63 \%)$ \\
Duration of Disease (months) & $3.7 \pm 1.7$
\end{tabular}


Table.2. Outcomes of Limberg flap reconstruction procedure

\begin{tabular}{lc}
\hline Outcome Variables & $\mathrm{n}=110$ \\
Mean duration of hospital stay (days) & $1.87 \pm 0.80$ \\
Mean postoperative pain score at 24 & 3.45 \\
hours after surgery & $95(86.36 \%)$ \\
Complete primary healing & $9(8.18 \%)$ \\
Surgical site Infection (n, \%) & $6(5.45 \%)$ \\
Seroma Formation (n,\%) & 0 \\
Flap necrosis & $2(1.81 \%)$ \\
Recurrence $(\mathrm{n}, \%)$ & \\
\hline
\end{tabular}

\section{DISCUSSION}

Natal cleft pilonidal sinus disease is known for recurrence and is quite problematic for patients both in terms of prolonged healing and added cost of treatment ${ }^{4}$. There is still conflict amongst surgeons about the ideal treatment for this disease. Various surgical procedures are in practice for this disease but a gold standard procedure has not been identified, because of high complication rate and high recurrence rate associated with all the procedures ${ }^{4}$. An ideal surgical procedure for this disease should have minimum complication rates, low cost, shorter duration of hospital stay and low recurrence rates ${ }^{4}$. Literature shows that to minimize the chances of recurrence, the natal cleft should be flattened and the closure of the wound should be away from the midline ${ }^{12,13}$. Surgical flap procedures which are done in the midline have more chances of wound dehiscence, wound infection and recurrence 4,9. Limberg flap reconstruction procedure provides a closure which flattens the natal cleft and closes the wound away from the midline which expedites the process of wound healing $3,4,14$. This is a large transposition flap based on a large vascularized pedicle ${ }^{14,15}$. This procedure has the advantages of being relatively easier to design and perform and the size of the flap can be customized according to the location and number of the pits in the natal cleft, creating a tension free wound and an acceptable scar ${ }^{14,21}$. Limberg flap is an excellent choice for patients who have complex pilonidal sinuses with multiple pits and for those who have had previous unsuccessful surgeries 15, 16. Post-operative drain placement is essential to avoid seroma formation and hence prevent wound infection. Slow return to normal daily activities, regular shaving of the hair from the nape of the neck and regular cleaning of the wound site are of prime importance to prevent recurrence ${ }^{3}$.

The results of this case series were comparable with the previous case series researches in terms of low surgical site infection rate, very low recurrence rate and no flap necrosis $^{3,4}, 14,16,17,18,19,20,21$. In this research only 9 patients $(8.18 \%)$ had a surgical site infection which resolved with oral antibiotics. 6 patients $(5.45 \%)$ had seroma formation which resolved by opening of the stitches at the affected site and no further surgical intervention was required. These results were better than some other studies. ${ }^{19,20}$ The length of hospital stay (1.87 \pm 0.80 days ) was also shorter than many other previous studies which is an advantage of this procedure, as it reduces the patients cost of hospital stay. ${ }^{17,18,19,20,21}$ The pain score calculated by VAS, 24 hours after the procedure was 3.45 , which falls in the moderate category score and was comparable to previous researches. ${ }^{21}$ No patient developed flap necrosis which was also better than some studies like Singh et.al and Chandu et.al. ${ }^{4,} 14$. Two patients $(1.81 \%)$ developed recurrence within six months of surgery and this recurrence rate was acceptable and comparable to other researches in the

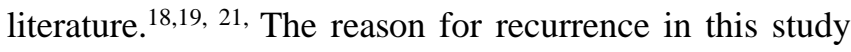
was that these patients didn't adhere to the long-term post-operative instructions and did not shave hair from the nape of the neck regularly. These hairs ultimately got impacted again in the natal cleft making a sinus again. Overall, $86 \%$ patients had complete primary healing which shows that this procedure is quite efficacious in treating primary natal cleft pilonidal sinus disease.

\section{STRENGTHS AND LIMITATIONS}

One of the strengths of this study was that a one-year follow-up time period was designated for each patient to observe the complications and any recurrence in this period. A limitation of this research was that Limberg flap reconstruction procedure was not compared with any other flap rotation procedure.

\section{CONCLUSION}

Limberg flap reconstruction procedure is a safe and efficacious surgical procedure for primary natal cleft pilonidal sinus disease because it provides early complete primary healing in most patients, and shortens the duration of hospital stay thus reducing the cost of treatment. The pain scores fall in the moderate postoperative pain score category and the complication rates and the recurrence rates are very low.

\section{CONFLICT OF INTEREST}

No conflict of interest was declared by the authors.

\section{FINANCIAL DISCLOSURE}

This study has not received any financial support from any external organization.

\section{ETHICAL APPROVAL}

The study was approved from Ethical Review Board of Lahore Medical and Dental College/ Ghurki trust 
teaching hospital, Lahore, Lahore, Pakistan, vide reference No. LMDC/03-2015, dated January 01, 2015.

\section{REFERENCES}

1. de Parades V, Bouchard D, Janier M, Berger A. Pilonidal sinus disease. J Visc Surg 2013;150(4):237-247.

2. Hull TL, Wu J. Pilonidal disease. Surg Clin North Am. 2002;82(6):1169-1185.

3. CN Y, Vijayakumar A. Limberg Flap Reconstruction for Pilonidal Sinus. International Journal of Biomedical and Advance Research. 2016;7(4): 165-168.

4. Singh PK, Gohil RK, Saxena N. Limberg flap procedure for sacrococcygeal pilonidal sinus: a prospective study. International Surgery Journal. 2017; 4(7):2238.

5. Karydakis GE. Easy And Successful Treatment Of Pilonidal Sinus After Explanation Of Its Causative Process. ANZ Journal of Surgery. 1992;62(5):385-389.

6. Surrell JA. Pilonidal disease. Surg Clin North Am. 1994;74:1309-1315.

7. Ates M, Dirican A, Sarac M, Aslan A, Colak C. Short and long-term results of the Karydakis flap versus the Limberg flap for treating pilonidal sinus disease: a prospective randomized study. The American Journal of Surgery. 2011;202(5):568-573.

8. Khatri VP, Espinosa MH, Amin AK. Management of recurrent pilonidal sinus by simple V-Y fasciocutaneous flap. Diseases of the Colon \& Rectum. 1994;37(12):1232-1235.

9. Bali I, Aziret M, Sözen S, Emir S, Erdem H, Çetinkünar S, et al. Effectiveness of Limberg and Karydakis flap in recurrent pilonidal sinus disease. Clinics. 2015;70(5):350-355.

10. Khan KJ, Ghaffar A, Choudhry S, Irshad K. Comparison of early outcome between modified Limberg and karydakis flap procedures in patients with sacrococcygeal pilonidal sinus. Pak J Med Health Sci 2016;10(2):631-634

11. Moran B, Hollingshead J, Farquharson M. Farquharsons textbook of operative general surgery. Boca Raton: Taylor \& Francis; 2016.

12. Petersen S, Koch R, Stelzner S, Wendlandt TP, Ludwig K. Primary closure techniques in chronic pilonidal sinus: a survey of the results of different surgical approaches. Dis Colon Rectum. 2002;45(11):1458-1467.
13. Mccallum I, King PM, Bruce J, Al-Khamis A. Healing by primary versus secondary intention after surgical treatment for pilonidal sinus. Cochrane Database of Systematic Reviews. 2007.

14. Chandu S, Y R. Limberg Flap Reconstruction For Pilonidal Sinus: Our Experience. Journal of Evolution of Medical and Dental Sciences. 2014;3(45):10986-10991.

15. Aithal SK, Rajan CS, Reddy N. Limberg Flap for Sacrococcygeal Pilonidal Sinus a Safe and Sound Procedure. Indian Journal of Surgery. 2012;75(4):298-301.

16. Urhan MK, Kücükel F, Topgul K, Özer I, Sari S. Rhomboid Excision and Limberg Flap for Managing Pilonidal Sinus. Diseases of the Colon \& Rectum. 2002;45(5):656-659.

17. Aslam MN, Shoaib S, Choudhry AM. Use of Limberg flap for pilonidal sinus-a viable option. J Ayub Med Coll Abbottabad. 2009 Oct-Dec; 21 (4): 31-33.

18. Mentes O, Bagci M, Bilgin T, Ozgul O, Ozdemir M. Limberg flap procedure for pilonidal sinus disease: results of 353 patients. Langenbecks Arch Surg. 2008 Mar; 393(2): 185-9. Epub 2007 Sep 22.

19. Akin M, Gokbayir H, Kilic K, Topgul K, Ozdemir E, Ferahkose Z. Rhomboid excision and Limberg flap for managing pilonidal sinus: long-term results in 411 patients. Colorectal Dis 2008; 10: 945-948

20. Katsoulis IE, Hibberts F, Carapeti EA. Outcome of treatment of primary and recurrent pilonidal sinus with Limberg flap. Surgeon. 2006;4(1):7-10.

21. Srihari RS, Naveen AM, Sreekar H. The limberg flap reconstruction - the optimal surgery for pilonidal sinus disease. Our Dermatology Online. 2016;7(3):271-275.

\section{AUTHOR'S CONTRIBUTIONS:}

WMC: Concept and Design, Data collection, Data analysis and Interpretation, Literature Search, Manuscript writing, Discussion

AS: Data collection, Literature Search, Manuscript writing:

MJ: Data collection, Literature Search, Discussion MSZ: Data analysis and Interpretation, Discussion ASB: Literature Search, Discussion MAD: Discussion 